1. A. M. TURING, "Rounding-off errors in matrix processes," Quart. Jour. Mech. and Appl. Math., v. 1, 1948, p. 289.

2. R. A. Frazer, W. J. Duncan, \& A. R. Collar, Elementary Matrices and Some Applications to Dynamics and Differential Equations, Cambridge Univ. Press, 1938, p. 99.

\title{
A Note on Large Linear Systems
}

Towards the end of 1955 the author of the present note was charged by Svenska Aeroplan Aktiebolaget (SAAB Aircraft Company), with setting up a program for the automatic computer BESK for the solution of large symmetric systems of linear algebraic equations. The reason was that the solution of 4 systems with 214 unknowns was required. The systems had been set up in conjunction with certain calculations for airplane structural analysis. Approximately $40 \%$ of the coefficients in the matrices of these systems differed from zero. This fact was taken into account in setting up the program. Nevertheless, the whole system could not be accommodated at one time in the magnetic drum memory of 8192 words associated with BESK, and a splicing method was developed which does not involve excessive loss of time in connection with the input and output of intermediate results. The four systems were solved simultaneously by the BESK. The method used for solving the equations was Gauss' method of elimination. The calculating time was about 2 hours. If $p \%$ of the coefficients of the systems differ from zero, and the order of the systems is $n$, the calculating time will be proportional to $p^{2} n^{3}$.

By formation of residuals and one iteration a solution was obtained which, when inserted into the original systems, proved to satisfy the equations with deviations less than $10^{-6} \%$, i.e., if the computed solution was $\bar{x}_{j}$ and the system was $A X=B$, the "error vector" $E=\sum_{j} a_{i j} \bar{x}_{j}-b_{i}$ satisfied $|E|<10^{-8}|B|$. The systems were badly conditioned since, if the matrix was $\left(a_{i k}\right)$, the "condition number" was:

$$
R(A)=\frac{\mid \text { determinant }\left(a_{i k}\right) \mid}{\prod_{k=1}^{214}\left(\sum_{i=1}^{214}\left|a_{i k}\right|\right)}=2^{-886}
$$

The matrix is therefore comparable with the $n \times n$ matrix

$$
A_{n}=\left[\begin{array}{rrrrrrrrr}
5 & -4 & 1 & . & . & . & & \\
-4 & 6 & -4 & 1 & . & . & & \\
1 & -4 & 6 & -4 & 1 & . & & \\
\cdot & 1 & -4 & 6 & -4 & 1 & & \\
& & & & . & & & & \\
& & & & & & & & \\
& & & & 1 & -4 & 6 & -4 & 1 \\
& & & & \cdot & 1 & -4 & 6 & -4 \\
& & & & . & . & 1 & -4 & 5
\end{array}\right]
$$


studied by Todd, for which $R\left(A_{n}\right) \sim c \cdot n^{2} \cdot 2^{-4 n}$ for a certain constant $c$. No direct comparison of the results of our experiments with those made by Todd [1] (for a $49 \times 49$ matrix, without iteration) is possible, since his results are expressed in terms of the norm of the error matrix.

The calculating program thus described operates at a fixed binary point and uses 40 binary digits. A program operating at a floating binary point and including 32 binary digits in its digital portion and 8 binary digits in its scalar exponent has also been developed. This program works at an approximately $30 \%$ slower rate and gave, when applied to the above-mentioned systems, a slightly better solution.

HANS RIESEL

\section{Essingeringen 9}

Stockholm

Sweden

1. J. ToDD, "The condition of certain matrices II," Arch. d. Math., v. 5, 1954, p. 249-257

\section{REVIEWS AND DESCRIPTIONS OF TABLES AND BOOKS}

65[A].-Robert L. Causey, Decimal to Octal and Octal to Decimal Conversion Tables, U. S. Naval Air Missile Test Center, Point Mugu, California, 1952, 30 p., $26.5 \mathrm{~cm}$. Deposited in the UMT FILE.

These are radix tables with three figure groups; when using the decimal to octal table the tabular entries are added together using octal arithmetic. The decimal to octal table has 11 place octal values of $n \times 10^{-K}$ for $n=0(1) 999$ and $K=3,6,9$. The octal to decimal table has 10 place decimal values of $n \times 8^{-K}$ for $n=0$ (1) 511 and $K=3,6,9,12$, together with an argument column giving $n \times 8^{-3}$ in octal. The tables were printed by photo-offset from output of an IBM tabulator. This machine has the unpleasant characteristic of leaving more space between adjacent columns than rows. The tables were developed for use with the RAYDAC.

University of California

J. L. Selfridge

Los Angeles, California

66[D, E].-Harvard University, Computation Laboratory, Annals, Tables of the Function arc $\sin z$, v. 40, Harvard University Press, Cambridge, Mass., 1956, xxxviii + 586 p., $27 \mathrm{~cm}$. Price $\$ 12.50$.

This table lists values of $w=\operatorname{arc} \sin z$, both the function $w$ and the argument $z$ being expressed in cartesian form. Symmetries make it unnecessary to consider values of $z$ belonging to points outside the first quadrant. If $w=u+i v$ and $z=x+i y$, both $u$ and $v$ are tabulated to 6 decimals for various values of $x$ and $y$. The results are arranged in 11 tables, in every one of which the lattice is square, that is, the intervals in $x$ and $y$ are equal. The intervals $\delta=\Delta x=\Delta y$ in Tables I to XI are respectively $0.002,0.005,0.01,0.02,0.05,0.1,0.2,0.5,1,2,5$. In a table of a function of a complex variable, the earlier intervals are strikingly small; previous tables of $\sin z$ and $\operatorname{arc} \sin z$ have rarely used intervals finer than 